\title{
Atuação Fonoaudiológica e Fisioterápica nas Fissuras Orofaciais não Sindrômicas
}

\section{Speech Therapy and Physiotherapy Performance in Non-Syndromic Orofacial Clefts}

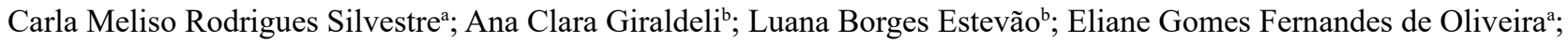 \\ Cristhiane Almeida Leite da Silva*c; Walkiria Shimoya-Bittencourt ${ }^{c}$ \\ ${ }^{a}$ Hospital Geral de Cuiabá, Departamento de Fissura Labiopalatinas. MT, Brasil. \\ bUniversidade de Cuiabá, Curso de Fisioterapia. MT, Brasil. \\ 'Universidade de Cuiabá, Programa de Pós-Graduação Stricto Sensu em Ciências Odontológicas Integradas. MT, Brasil. \\ *E-mail:cristhianeleite@hotmail.com
}

\begin{abstract}
Resumo
A fissura de lábio e ou de palato não sindrômicas são anomalias congênitas craniofaciais mais frequentes. Elas ocasionam problemas estéticos e funcionais que requerem tratamento em longo prazo, envolvendo reabilitação multidisciplinar incluindo a fonoaudiologia e fisioterapia. Este estudo propõem apresentar alguns dos princípios de atuação do fonoaudiólogo e do fisioterapeuta nas fissuras orofaciais não sindrômicas. Realizou-se uma revisão de literatura narrativa com busca na Bireme e Scielo e nas bases Lilacs, Pedro e PubMed em outubro de 2020, envolvendo a atuação do fonoaudiólogo e do fisioterapeuta nas fissuras orofaciais não sindrômica, no idioma inglês e português, sem recorte temporal. Os estudos encontrados observaram que a intervenção fonoaudiológica e fisioterapêutica deve ser mais precoce e de acordo com a disfunção apresentada. De modo geral, a atuação do fonoaudiólogo favorece a alimentação oral e o desenvolvimento global referente à linguagem, a fala, audição e neuropsicomotor, para evitar atrasos e favorecer o melhor desenvolvimento infantil. A atuação fisioterapêutica visa diminuir a hospitalização prolongada, melhorar a qualidade de vida e funcionalidade, bem como assistir as crianças que cursarem com problemas motores, posturais e respiratórios. Conclui-se que a atuação fonoaudiológica nas diferentes fases da reabilitação de indivíduos com fissuras labiopalatinas contribui para alimentação e inteligibilidade da fala, beneficiando assim a comunicação verbal e consequentemente a interação com o meio social; e a assistência fisioterapêutica ajuda e melhorar a sintomatologia e as disfunções respiratórias apresentadas, prevenindo e tratando complicações de forma a melhorar a qualidade de vida e restabelecer a independência funcional.
\end{abstract}

Palavras-chave: Fissura Palatina. Fenda Labial. Aleitamento Materno. Fala. Fisioterapia.

\begin{abstract}
Non-syndromic cleft lip and or palate are the most frequent congenital craniofacial anomalies. They cause aesthetic and functional problems that require long-term treatment, involving rehabilitation including speech therapy and physiotherapy. This study proposes to present some of the principles of performance of the speech therapist and physiotherapist in non-syndromic orofacial clefts. A narrative literature review was carried out with searches in Bireme and Scielo and in the Lilacs, Pedro and PubMed databases in October 2020, involving the performance of the speech therapist and physiotherapist in non-syndromic orofacial clefts, in English and Portuguese, with no time frame. The studies observed that speech therapy and physiotherapy intervention should be as earlier and in accordance with the presented dysfunction. In general, the performance of the speech therapist favors oral feeding and the global development related to language, speech, hearing and neuropsychomotor, to avoid delays and favor best child development. Physiotherapeutic action aims to reduce the prolonged hospitalization, improve quality of life and functionality, as well as assist children who are experiencing motor, postural and respiratory problems. It is concluded that the speech therapy performance in the different phases of rehabilitation of individuals with cleft lip and palate contributes to feeding and speech intelligibility, thus benefiting verbal communication and consequently the interaction with the social environment; and physiotherapeutic assistance helps and improves the symptoms and respiratory disorders presented, preventing and treating complications in order to improve the quality of life and restore functional independence.
\end{abstract}

Keywords: Cleft Palate. Cleft Lip. Breast Feeding. Speech. Physiotherapy.

\section{Introdução}

A fissura de lábio e ou de palato não sindrômicas são anomalias congênitas craniofaciais mais frequentes entre os humanos, acometendo de 1 a 2 crianças em cada 1000 nascimentos no mundo(LIDRAL, 2008; CYMROT etal., 2010; FREITAS et al. 2012). A literatura refere à multifatoriedade em relação às etiologias das fissuras orofaciais, relacionadas principalmente as exposições maternas durante o primeiro trimestre gestacional, período esse, onde ocorre a formação da face da criança, resultando em falha da fusão do lábio e palato (FERGUSON, 1988; LEBBY, TAN, BROWN, 2010;
WEHBY, 2013; SKULADOTTIR, 2014; SPINDER et al., 2017; DUTRA, FERREIRA, 2019).

Fatores genéticos, interações poligênicas, tabagismo, etilismo, uso de medicamentos, carência de vitaminas, irradiações e exposições à produtos químicos são descritos na literatura como fatores contribuintes para ocorrência dessa malformação. Essas malformações alteram a anatomia normal, podendo interferir na fala, na audição, na deglutição, na respiração e na dentição (MATTOS et al., 2007; WEHBY, 2013; SKULADOTTIR et al., 2014; LEBBY; TAN; BROWN, 2010; SPINDER et al., 2017; DUTRA; FERREIRA, 2017).

Os problemas estéticos e funcionais causados pelas fissuras 
requerem tratamento de longo prazo, envolvendo cirurgias reparadoras e reabilitação com equipe multidisciplinar envolvendo dentre as áreas profissionais a cirurgia plástica, pediatria, psicologia, odontologia, otorrinolaringologia, nutrição, fonoaudiologia e fisioterapia (ALMEIDA et al., 2017, PEREIRA, 2019). A reabilitação completa dos pacientes com fissuras labiopalatinas é a meta almejada por toda equipe. Cada profissional através de uma avaliação específica estabelece o seu segmento pré e pós-cirúrgico, em consonância com o planejamento da equipe (RIBEIRO, SABÓIA, FONTELES, 2011).

Portanto, o objetivo deste estudo foi apresentar alguns dos princípios de atuação do profissional fonoaudiólogo e fisioterapeuta nas fissuras orofaciais não sindrômicas.

\section{Desenvolvimento}

\subsection{Metodologia}

Realizou-se uma revisão narrativa de literatura para conhecer e sintetizar os conhecimentos sobre determinada temática. A busca foi realizada nas bases bibliográficas PubMed, Bireme, Scielo, Lilacs e Pedro envolvendo a atuação do profissional fonoaudiólogo e fisioterapeuta nas fissuras orofaciais não sindrômicas, utilizando unitermos em inglês e português associando com o operador booleano AND. Para a fonoaudiologia os descritores utilizados foram: Cleft palate, Cleft Lip, Breast feeding, Speech; e para a fisioterapia utilizou-se as seguintes palavras-chaves: fenda labial, lábio leporino, reabilitação e fisioterapia.

A busca ocorreu no mês de outubro de 2020 pelos pesquisadores do estudo. Incluíram-se artigos nos idiomas inglês e português publicado em periódicos nacionais e internacionais acerca da temática da pesquisa, sem restrição quanto ao período de publicação. Excluíram-se os artigos que não possuíam conteúdo concernente com o pesquisado, resumos de anais e cartas ao editor. Apontamentos de livros, trabalhos, manuais, dissertações e teses com a mesma temática também foram considerados.

\subsection{Atuação da Fonoaudiologia}

A atuação fonoaudiológica será dividida para fins didáticos em três fases: do nascimento aos 3 anos de idade, a partir dos 4 anos de idade e fase adulta.

\subsubsection{Do nascimento aos 3 anos de idade}

A intervenção deve ser a mais precoce possível, desde a maternidade, sendo que a presença da fonoaudiologia nas equipes de neonatologia tem favorecido a alimentação oral (D’Agostino, 2004; DIOGO, 2012). Dessa maneira, o incentivo ao aleitamento materno deve ser priorizado para todas as crianças pelo benefício imunológico, nutricional, afetivo e psicológico (LOFIEGO, 1992; MELENDEZ, 2004; ROCHA et al., 2008).

No caso de bebês com fissuras labiais as maiores dificuldades encontradas são em relação à pega ao seio materno e ao posicionamento para beneficiar a amamentação (BRANCO, CARDOSO, 2013; SANTOS; JANINI; OLIVEIRA, 2019). Já nos casos onde o palato foi acometido pela fissura, as dificuldades na sucção se referem à diminuição da pressão intraoral em virtude da comunicação entre a cavidade oral e nasal, diminuindo assim a força da sucção necessária para extração do leite, podendo contribuir no baixo peso ponderal, devido ao gasto energético (ALTMANN et al., 1997, MONTAGNOLLI et al., 2005; REID, KILPATRICK, REILL, 2006; CAMPILLAY, DELGADO, BRESCOVICI, 2009; DI NINNO et al., 2011; GENARO, MODOLO, MIGUEL, 2012).

A atuação fonoaudiológica nessas crianças é para auxiliar as nutrizes e orientar as mesmas quanto ao posicionamento elevado, semissentado, minimizando a ocorrência de refluxo do leite para cavidade nasal, bem como possível risco de líquido à orelha média, que são comuns e frequentes nesses bebês devido à disfunção velofaríngea e da tuba auditiva (LOFIEGO, 1992; ALTMANN et al., 1997; GOLDINGKUSHNER, 2001; CARRARO; DORNELLES;COLLARES, 2011; BRANCO, CARDOSO, 2013; WEYAND, BARBOSA, 2017; LEVY, ALMEIDA, 2018).

Quando o aleitamento materno não é possível, devido ao distúrbio do sistema sensório motor oral, é indicada a ordenha do leite materno com uso de mamadeiras, seja bicos ortodônticos, bicos universais ou específicos para essa malformação. A literatura reforça que o bico mais adequado é o que a criança melhor se adaptar e que favoreça a saúde dela, contribuindo para que as cirurgias reparadoras ocorram na época preconizada. (ALTMANN et al. 1997; REID, KILPATRICK, REILL, 2006, CARRARO; DORNELLES; COLLARES, 2011; SANTOS et al. 2011; WEYAND; BARBOSA, 2017; LEVY, ALMEIDA, 2018; SANTOS, JANINE, OLIVEIRA, 2019).

Em alguns centros de referência em atendimento de pacientes com fissuras labiopalatinas, a partir dos 3 meses realiza-se as cirurgias de queiloplastias (cirurgia para correção labial), preconizando nesse momento a suspensão de uso de mamadeiras, e chupetas que exijam os movimentos de sucção, evitando assim a abertura da sutura cirúrgica. No pós-operatório é indicada a substituição por mamadeiras de colher e/ou copinho até a liberação pós-cirúrgica do cirurgião responsável (ALTMANN et al., 1997; FREITAS, 1998; OLIVEIRA, 2018; DI NINNO, JESUS, 2009; SANTOS et al., 2011; GENARO, MODOLO, MIGUEL, 2012).

Após 30 dias da queiloplastia, é orientado aos pais e ou responsáveis realizarem estimulações orofaciais através de massagens extras e intra orais sobre a cicatriz cirúrgica com movimentos verticais, horizontais e circulares em musculatura perioral, visando melhor resultados estéticos e funcionais (ALTMANN, 1997; ROSSI et al., 2005; DI NINNO; JESUS, 2009; MONLLEÓ, 2014). 
O fonoaudiólogo acompanha e orienta ainda, sobre o desenvolvimento global nos aspectos referentes a linguagem, audição e neuropsicomotor, a fim de evitar atrasos e favorecer melhor o desenvolvimento infantil (GENARO, 2004; GENARO, FELICIO, 2014).

$\mathrm{Na}$ fase do balbucio em casos de crianças com fissuras palatinas ainda não corrigidas, é orientado aos pais a estimulação sensitiva através da oclusão nasal com objetivo de propriocepção do direcionamento do fluxo aéreo para cavidade oral. (PEGORARO-KROOK et al., 2004; DUTKASOUZA, NEVES, PEGORARO-KROOK, 2007; GENARO, MODOLO; MIGUEL, 2012; HORTIS-DZIERZBICKA, RADKOWSKA, FUDALEJ et al.,2012; MILDINHALL, 2012). A partir de 15 meses é efetivada a correção da palatoplastia (correção do palato primário e secundário) onde além dos aspectos citados anteriormente, a intervenção fonoaudiológica visa prevenir as instalações de articulações compensatórias na fala (GOLDING-KUSHNER, 2001; PEGORARO-KROOK et al. 2004; GENARO, MODOLO, MIGUEL, 2012; MILDINHALL, 2012).

Desde a aquisição do inventário fonético-fonológico, após palatoplastia são realizadas avaliações periódicas dos 3 aos 4 anos, com o intuito de saber se a criança apresenta trocas fonológicas do próprio desenvolvimento ou distúrbios fonéticos ou fonológicos, assim como podem apresentar após palatoplastia, disfunção velofaríngea, regurgitação nasal de alimentos, escape de ar nasal, fraca pressão intraoral, distúrbio articulatórios compensatórios e hipernasalidade na fala, necessitando assim de fonoterapia (GOLDING-KUSHNER, 2001; PEGORARO-KROOK et al., 2004; PETERSON FALZONE et al., 2006; PALANDI, GUEDES, 2011; GENARO, MODOLO, MIGUEL 2012; MILDINHALL, 2012; KUMMER, 2016).

\subsubsection{A partir dos 4 anos de idade}

Aos 4 anos de idade em pacientes que mesmo após a palatoplastia há uma falha no fechamento velofaríngeo devido á incompetência velar, a fonoterapia visa o direcionamento do fluxo aéreo para cavidade oral, eliminar ou amenizar a hipernasalidade e as articulatórias compensatórias presentes (GOLDING-KUSHNER,2001; BRUNNEGARD, LOHMANDER, 2007; GENARO; MODOLO; MIGUEL, 2012; MARTINS, CARDOSO, 2015; KUMMER, 2016).

Nos casos em que as crianças apresentam distúrbios fonológicos, que são alterações na produção dos fonemas da fala, a fonoterapia é direcionada para adequar o ponto e modo articulatório do fonema, através de pistas multissensoriais (GENARO, 2004; MARTINS, CARDOSO, 2015).

\subsubsection{Adultos}

Nos adultos com discrepâncias esqueléticas e dentárias é indicada a cirurgia ortognática, onde a atuação fonoaudiológica pré-operatória é direcionada para conscientização e/ou para eliminação ou minimização dos hábitos deletéricos e no pósoperatório para organização e adequação da musculatura orofacial e das funções do sistema estomatognático, a fim de evitar recidivas (SILVA FILHO et al., 1998; D'AGOSTINO, 2004; BERRETIM-FELIX, JORGE, GENARO, 2004; LOHMANDER, PERSSON, 2008; PAULUS, 2014).

\subsection{Atuação da Fisioterapia}

A atuação fisioterapêutica nas fissuras labiopalatinas pode ser compreendida em termos ambulatoriais e de internações, abrangendo o período pré e pós-operatório. As técnicas realizadas tem o objetivo de diminuir a hospitalização prolongada do paciente como também de melhorar a qualidade de vida e funcionalidade, bem como assistir as crianças que cursarem com problemas motores, posturais e respiratórios (ALMEIDA et al., 2017).

\subsubsection{Período pré-operatório}

As ações desenvolvidas pela fisioterapia no período préoperatório consistem em fornecer informações adequadas ao paciente e responsáveis sobre os procedimentos de avaliação e tratamento, orientar sobre os possíveis sintomas que podem ocorrer após o procedimento cirúrgico e incentivar a participação dos responsáveis no processo de tratamento (FERNANDES; DEFANI, 2013). As orientações sobre a respiração, postura e amamentação para que o paciente possa se alimentar são imprescindíveis.

Faz parte da conduta educar a respiração do paciente, tanto para que o indivíduo respire da maneira mais adequada possível mesmo com o palato fissurado quanto para preparar para o ato cirúrgico e evitar complicações pulmonares, mediante a realização de exercícios respiratórios de inspirações e expirações forçadas e fracionadas (ALMEIDA, 2017). Como também, atuar no preparo da mama da mãe e orientar a pega correta desse seio, favorecendo o início da lactação, uma vez que a ingestão de nutrientes deve ser garantida para proporcionar o crescimento e ganho de peso necessário, e dessa forma, evitando complicações no pósoperatório (FREITAS; CARDOSO, 2019).

Devido às alterações anatômicas e estruturais de lábio, alvéolo, palato duro e palato mole, há o favorecimento de refluxo do alimento para a cavidade nasal. Tal fato associado à presença de escape nasal, sintoma de disfagia frequentemente referido, pode levar a dificuldade de realizar tosse voluntária, acúmulo de secreção e alimentos, favorecendo a pneumonia e outras patologias aumentando o risco de complicações e hospitalização. Torna-se essencial utilizar recursos de fisioterapia respiratória como técnicas de higiene brônquica, fortalecimento muscular e reeducação da tosse com vistas a melhorar as disfunções apresentadas (FREITAS; CARDOSO, 2018). 


\subsubsection{Período pós-operatório}

É comum paciente com fissura realizarem a cirurgia ortognática como correção da deformidade e como parte do processo de reabilitação. A assistência fisioterapêutica é principalmente voltada para a sintomatologia e disfunções apresentadas no pós-operatório, como por exemplo, edema facial, alteração respiratória, dificuldade de tossir, fraqueza muscular, dentre outros. A realização da técnica de drenagem linfática manual facial é frequentemente utilizada para diminuição de edema (GODOY; GODOY, 2004).

A Drenagem linfática Manual (DLM) é um recurso específico da área de massoterapia, cuja maior função é esvaziar os excedentes de líquidos e as sobras metabólicas por meio de ações nos linfonodos e nas vias linfáticas (JUNIOR, 2001). A DLM é bastante utilizada no tratamento de várias doenças, pois age no sistema circulatório linfático (linfonodos, vasos linfáticos e linfa) propiciando diminuição do inchaço (GODOY, 2010). A técnica vai ajudar na ampliação do transporte de linfa, assim, otimizando a vascularização, a anastomose linfolinfática e linfovenosa e possibilitar aumento da resistência imunitária do corpo, causado pelo maior número de células imunes que circulam pelo sistema linfático (LEDUC, 2012). O rosto pode ser drenado manualmente de 3 a 5 vezes em cada local ou quanto achar necessário, sendo extremamente importante evitar a região próxima a boca e nariz, local do procedimento cirúrgico (OLIVEIRA, 2015).

Com o intuito de reestabelecer a respiração, podemse utilizar técnicas de fisioterapia respiratória mediante a realização de exames de avaliação da capacidade e força muscular respiratória (ANTUNES, 2013). Poderão ser usadas técnicas de higiene brônquica com vistas à mobilização e a eliminação de secreções nas vias respiratórias através do aumento do fluxo expiratório entre outras (BRITO, 2014).

A orientação quanto ao posicionamento do paciente também será muito importante na reabilitação. A posição de decúbito lateral ou dorsal com a cabeceira elevada vai ajudar a evitar possíveis traumas que possam vir a acontecer nos locais sensíveis da cirurgia, além de facilitar a respiração, tosse e manuseio do paciente. Ademais, promovendo uma recuperação mais segura e rápida (LOPES, 2013).

Em relação a alterações motoras que podem ocorrer, a fisioterapia vai atuar na avaliação e acompanhamento do desenvolvimento neuropsicomotor desse paciente. O fisioterapeuta vai promover estímulos neuromusculares através de técnicas neuromusculares e proprioceptivas para aquisição ou melhora das habilidades motoras. A avaliação pós-cirúrgica será extremamente importante, uma vez que os procedimentos cirúrgicos invasivos podem influenciar diretamente no desenvolvimento esperado para a idade da criança (LOPES, 2013).

\section{Conclusão}

Os princípios da atuação fonoaudiológica se baseiam nas diferentes fases da reabilitação de indivíduos com fissuras labiopalatinas com vistas a contribuir na melhoria da qualidade de vida, alimentação e inteligibilidade da fala, beneficiando assim a comunicação verbal e consequentemente a interação com o meio social. A assistência fisioterapêutica desenvolvida fundamenta-se nos períodos pré e pós-operatório com o intuito de melhorar a sintomatologia e as disfunções respiratórias apresentadas, prevenindo e tratando complicações de forma a melhorar a qualidade de vida e restabelecer a independência funcional.

Como parte do processo de reabilitação, torna-se imprescindível a inserção do fonoaudiólogo e fisioterapeuta na equipe de saúde, uma vez que a expertise de cada profissional contribui para recuperação da funcionalidade prejudicada. Dessa forma, se faz necessários mais estudos para aprimorar e inovar os métodos de atuação do fonoaudiólogo e fisioterapeuta nas fissuras orofaciais não sindrômicas.

\section{Referências}

ALMEIDA, A.M.F.L. et al. Atenção à pessoa com fissura labiopalatina: proposta de modelização para avaliação de centros especializados no Brasil. Saúde Debate.v.41, p.156-166, 2017. doi: 10.1590/0103-11042017S12

ALTMANN, E.B.C. et al. Tratamento fonoaudiológico. In ALTMANN, E.B.C. Fissuras labiopalatinas. Carapicuíba: Pró Fono, 1997. p.291-324.

ALTMANN, E.B.C., et al. Tratamento precoce. In: ALTMANN, E.B.C. Fissuras labiopalatinas. Carapicuíba: Pró Fono, 1997. p. 291-324.

ANTUNES V.L. et al. Fisioterapia respiratória em pacientes com fissuras labiopalatinas: rotinas do Hospital de Reabilitação de Anomalias Craniofaciais. Anais. 2013.

BERRETIM-FELIX, G.; JORGE, T.M.; GENARO, K.F. Intervenção fonoaudiológica em pacientes submetidos à cirurgia ortognática. In: FERREIRA, L.P.; LOPES-BEFI, D.M.; LIMONGI, S.C.O. Tratado de fonoaudiologia. São Paulo: Roca, 2004. p.494-511.

BRANCO, L.L.; CARDOSO, M.C. Alimentação no recémnascido em fissuras labiopalatinas. Universitas: Ciênc. Saúde, v.11, n.1, p.57-70, 2013. doi: 10.5102/UCS.V11/1.1986

BRITO, R.R; BRANT, T.C.S; PARREIRA, V.F. Recursos manuais e instrumentais em fisioterapia respiratória. São Paulo: Manole, 2014.

BRUNNEGARD, K.; LOHMANDER, A. A cross: sectional study of speech in 10- year-old children with Cleft palate: results and issues of rater reliability. Cleft Palate Crânio J., v.44, , p.3344, 2007. doi: 10.1597/05-164

CAMPILLAY P.L.; DELGADO S.E.; BRESCOVICI, S.M. Avaliação da alimentação em crianças com fissura de lábio e/ou palato atendidas em um hospital de Porto Alegre. Rev. CEFAC, v.12, n.2, p. 257-266, 2010. doi: 10.1590/S151618462010005000010 .

CARRARO, D.F., DORNELLES, C.T.L., COLLARES, M.V.M. Fissuras labiopalatinas e nutrição. Rev HCPA, v.31, n.4, p.456-63, 2011.

CYMROT, M., et al. Prevalência dos tipos de fissura em pacientes com fissuras labiopalatinas atendidos em um Hospital Pediátrico do Nordeste brasileiro. Revista Brasileira de Cirurgia 
Plástica, v. 25, n. 4, p. 648-651, 2010. doi:10.1590/S198351752010000400015

D'AGOSTINO, L. Fissuras labiopalatais: o que mudou no tratamento? In: Comitê de Motricidade Orofacial - SBFa. Motricidade orofacial: como atuam os especialistas. São Paulo: Pulso, p. 203-209, 2004.

DI NINNO, C.Q.M.S.; JESUS, M.S.V. Terapia fonoaudiológica para alterações de fala decorrentes de fissura labiopalatina. In: JESUS, M.S.V.; DI NINNO, C.Q.M.S. Fissura labiopalatina: fundamentos para a prática fonoaudiológica. São Paulo: Roca. p.76-98, 2009.

DI NINNO, C.Q.M.S., et al. Aleitamento materno exclusivo em bebês com fissura de lábio e/ou palato. Rev. Soc. Bras. Fonoaudiol., v.16, n.4, p.417-421, 2011. doi: 10.1590/S151680342011000400009

DIOGO, J.R.M. Tratamento fonoaudiológico precoce em crianças com fissuras lábio palatinas. Rev. Saúde e Beleza. Ceará. 2012.

DUTKA-SOUZA, J.; NEVES, J.F.D.A.; PEGORARO-KROOK, M.I. "Soquinhos" ou "raspadinhos"? Do que estamos falando? Informativo aos pais e cuidadores. Bauru: HRAC: USP, 2007. doi: 10.1590/0103-1104201912108

FERGUSON, M. W. J. Palate development. Development 103 Supplement, p. 41-60, 1988.

FERNANDES, R; DEFANI, M.A. Importância da equipe multidisciplinar no tratamento e proservação de fissuras labiopalatinas. Rev Saúde Pesq., v. 6, n.1, p.109-116, 2013.

FREITAS, J.S; CARDOSO, M.C.A.F. Sintomas de disfagia em crianças com fissura labial elou palatina pré e pós-correção cirúrgica. Codas. v.30, n.1, p.1-7, 2018. doi: 10.1590/2317$1782 / 20182017018$

FREITAS, M. Q. Tratamento fonoaudiológico das fissuras labiopalatinas. In: JUNQUEIRA, P.; DAUDEN, A.T.B. (Org.) Aspectos atuais em terapia fonoaudiológica: deglutição, articulação, leitura/escrita, voz, linguagem. São Paulo: Pancast, 1998. p.25-38.

FREITAS, J.A.S. et al. Rehabilitative treatment of cleft lip and palate: experience of the Hospital for Rehabilitation of Craniofacial Anomalies/USP (HRAC/USP) - Part 1: overall aspects. J. Appl. Oral Scie., v.13, p.9-15, 2012.

GENARO, K. F. A terapia fonoaudiológica para a correção da produção da fala nos casos com fissura labiopalatinas já reparada. In: COMITE DE MOTRICIDADE OROFACIAL - SBFa. Motricidade orofacial: como atuam os especialistas. São Paulo: Pulso, 2004. p.211-219.

GENARO, K.F.; MODOLO, D.J.; MIGUEL, H.C. Fissura labiopalatina. In: PERNAMBUCO, L.A. et al. Atualidades em motricidade orofacial. Rio de Janeiro: Revinter, 2012. p. 149161 .

GENARO, K.; FELICIO, C.M. Protocolos clínicos de avaliação miofuncional orofacial. In: MARCHESAN, I., SILVA H., THOMÉ, M. Tratado de fonoaudiologia. São Paulo: Roca, 2014. p.271-282,

GODOY, J.M.P.; GODOY, M.F.G. Drenagem linfática manual: novo conceito. J. Vasc. Bras., v.3, n.1, p.77-80, 2004.

GODOY, J. M.P.; GODOY, M.F.G. Drenagem linfática manual: uma nova abordagem. São Paulo: Lincomunicações, 2010.

GOLDING-KUSHNER, K.J. Therapy Techniques for cleft palate speech and related disorders. San Diego: Singular Publishing, 2001.

HORTIS-DZIERZBICKA, M.; RADKOWSKA, E.; FUDALEJ,
P. Speech outcomes in 10-year-old children with complete unilateral cleft lip and palate after onestage lip and palate repair in the first year of life. J. Plast. Reconstr Aesthet. Surg., v.65, n.2, p.175-81, 2012. doi: 10.1016/j.bjps.2011.09.015

JUNIOR, R. Estética e cirurgia plástica tratamento no pré e pós operatório. São Paulo: Senac, 2001

KUMMER, A.W. Evaluation of speech and resonance for children with craniofacial facial Plast Surg. Clin. N. Am., v.24 p.445-451 2016. doi:/10.1016/j.fsc.2016.06.003

LEBBY, K.D.; TAN, F.; BROWN, C.P. Maternal factors and disparities associated with oral clefts. Ethnicity Dis., v.20, n.1, p.S1-146-9, 2010.

LEDUC, A.; LEDUC, O. Drenagem linfática: teoria e prática. São Paulo: Manole, 2012.

LIDRAL, A.C; MORENO, L.M.; BULLAR, S.A. Genetic factors and orofacial clefting. Seminars in Orthodontics, v.14, n.2, p.103114, 2008. doi: 10.1053/j.sodo.2008.02.002

LOFIEGO, J.L. Fissura lábio palatino: avaliação, diagnóstico e tratamento fonoaudiológico. Rio de Janeiro. Revinter,1992.

LOPES, I. M. B.; ARRUDA, K. A. Atuação da fisioterapia na reabilitação de pacientes com anomalias craniofaciais. Bauru: Universidade de São Paulo, Hospital de Reabilitação de Anomalias Craniofaciais, 2013.

LOHMANDER, A., PERSSON, C. A longitudinal study of speech production in swedish children with unilateral cleft lip and palate and two-stage palatal repair. Cleft Palate Carniofac J., v.45, p.32-41, 2008.

MATTOS, B.S.C.; MAURO, L.D.V. Fissuras labiopalatinas: interdisciplinaridade e conceitos atuais em odontologia. In: HADDAD, A.S. Odontologia para pacientes com necessidades especiais. São Paulo, 2007. p.196-205,

MARTINS, P.B., CARDOSO F.A.C.M. Variações articulatórias nas fissuras labiopalatinas com enfoque fonoterapêutico. Universitas, Ciências da saúde. V.13. n.1, Brasilia, 2015.

MELENDEZ-VELASQUES, G. Aleitamento materno exclusivo na alta de recém-nascidos internados em berçário de alto risco e os fatores associados a essa prática. J. Pediatr., v.80, n.3, p.33-37, 2004.

MILDINHALL, S. Speech and language in the patient with cleft palate. Front Oral Biol., v.16, p.137-146, 2012.

MONLLEÓ, I.L, MENDES, L.G.A., LOPES, V.L.G.S. Manual de cuidados de saúde e alimentação da criança com fenda oral. Projeto crânio-face Brasil, v. 22, n.5, p. 913-921, 2014.

MONTAGNOLLI, L.C. et al. Prejuízo no crescimento de crianças com diferentes tipos de fissuras lábio-palatina nos 2 primeiros anos de idade. J. Pediatr., v.81,p.461-465, 2005.

OLIVEIRA, R.P. Abordagem fonoaudiológicas disfagias em malformações craniofaciais. In: LEVY, D.S. Disfagia infantil. Rio de Janeiro: Thieme Revinter 2018. p.155-160.

OLIVEIRA, K.C. O Beneficio da fisioterapia utilizando a drenagem linfática manual facial no pós-operatório de Bleferoplastia. Disponível em: <http://www.portalbiocursos. com.br/artigos/dermfuncional/19.pdf> acesso em: 9 nov, 2020

PAULUS, C. Orthognathic surgery for patients with cleft lip and palate. Rev. Stomatol Chir Maxillofac Chir Orale., v.115. n.4, p.239-244, 2014.

PALANDI, B.B.B.; GUEDES, Z.C.F. Aspecto de fala de indivíduos com fissura palatina e labial, corrigida em diferentes idades. Rev CEFAC., v.13, n.1, p.8- 16, 2011. 
PEGORARO-KROOK, M. I., et al. Intervenção fonoaudiológica na fissura palatina. In: FERREIRA, L.P.; BEFI-LOPES, D.M.; LIMONGI, S.C.O. (Org.) Tratado de fonoaudiologia. São Paulo: Rocca, 2004. p.439-455,

PEREIRA, B.G. A multidisciplinaridade em fissuras labiopalatinas. Rev. Cient. Multidisc. UNIFLU, v.4, n.2, 2019.

PETERSON-FALZONE, S.J. et al. The clinican's guide to treating cleft palate speech. St. Louis: Mosby Effects of cleft and non-cleft VPI on speech in older children, 2006.

REID, J.B.; KILPATRICK, N.; REILL.Y.S. A prospective longitudinal study of feeding skills ia a cohort of babies with cleft conditions. Cleft Palate-Craniofac. J., v.43, n.6, p.702-709, 2006. doi: 10.1597/05-172

RIBEIRO, T.R.; SABÓIA, V.P.A.; FONTELES, C.S.R. Fissuras labiopalatais: abordagem multiprofissional. Bras. Med., v.48. n.3, p.290-295, 2011

ROCHA, C.M.G. et al. Aleitamento materno e fissura labiopalatal: revisão e atualização. Rev. Med. Minas Gerais, v.18, n.4, p.77-82, 2008.

ROSSI, D. C.; et al. O efeito da massagem no processo de cicatrização labial em crianças operadas de fissura transforame unilateral. Rev. CEFAC, v.7, n.2, p.205-214, 2005.

SANTOS, E.C., et al. Análise qualitativa do padrão alimentar de crianças portadoras de fissuras de lábio e/ou palato atendidas em um hospital de Goiânia-GO. J. Health Sci. Inst. Goiânia. 2011.
SANTOS, L.R.B. Atuação fonoaudiológica na amamentação de bebês com fissura palatina. Rev. Cient. Multidisc. UNIFLU, v.4, n.2, 2019 .

SANTOS, S.R.; JANINI P.J.; OLIVEIRA S.M.H. A transição na amamentação de crianças com fenda labial e palatina. Rev. Esc. Anna Nery. v.23 n.1, 2019. doi: 10.1590/2177-9465-EAN-20180152

SILVA FILHO, O.G. et al. A cirurgia ortognática na reabilitação do paciente portador de fissura unilateral completa de lábio e palato. Rev. Dental Press Ortodon. Ortop. Facial, v.3, n.4, p.5170, 1998 .

SKULADOTTIR, $\mathrm{H}$. et al. First-trimester non-systemic corticosteroid use and the risk of oral clefts in Norway. Ann. Epidemiol, v.24, n.9, p.635-640, 2014. doi: 10.1016/j. annepidem.2014.06.005

SPINDER, N. et al. Maternal occupational exposure and oral clefts in offspring. Environ. Health, v.6, n. 83, 2017. doi: 10.1186/ s12940-017-0294-5

WEHBY, G.L. Avançando e priorizando a pesquisa sobre fissuras orais no Brasil. J. Pediatr., v.89, n.2, p.112-115, 2013. doi: 10.1016/j.jped.2013.03.010

WEYAND, I.C.G.; BARBOSA, D.A. Como o meu bebê vai mamar? In: BARBOSA, D.A.; PANNUNZIO, L. As fissuradas: guia de orientações sobre fissura labiopalatina. Ribeirão Preto: Book Toy, 2017. 